\title{
Cold Reactive Collisions between Laser-Cooled Ions and Velocity-Selected Neutral Molecules
}

\author{
Stefan Willitsch, ${ }^{*}$ Martin T. Bell, Alexander D. Gingell, Simon R. Procter, and Timothy P. Softley ${ }^{\dagger}$ \\ Department of Chemistry, University of Oxford, Chemistry Research Laboratory, Oxford OX1 3TA, United Kingdom
}

(Received 15 August 2007; published 30 January 2008)

\begin{abstract}
We report a new experimental method to study reactive ion-molecule collisions at very low temperatures. A source of laser-cooled ions in a linear Paul trap has been combined with a quadrupole-guide velocity selector to investigate the reaction of $\mathrm{Ca}^{+}$with $\mathrm{CH}_{3} \mathrm{~F}$ at collision energies $\bar{E}_{\text {coll }} / k_{B} \geq 1 \mathrm{~K}$ with single-particle sensitivity. The technique represents a general approach to study reactive collisions between ions and polar molecules over a wide temperature range down to the cold regime.
\end{abstract}

PACS numbers: 34.50.Lf, 37.10.De, 82.30.Fi

The recent development of a range of techniques for producing "cold" molecules at very low translational temperatures, $T \leq 1 \mathrm{~K}$, has provided the opportunity for studying gas-phase collisional processes in a new physical regime in which the de Broglie wavelength of the interacting particles is comparable with, or longer than, the scale of molecular interactions. Recent theoretical studies of the dynamics of reactive molecular-scattering processes at very low energies show that these may be dominated by severe constraints on the orbital angular momentum of the collision, the occurrence of reactive resonances and tunneling, and a pronounced dependence of the cross section on the collision energy [1-3]. The low-temperature regime represents a unique environment for studying the quantummechanical details of chemical reactions which are often averaged out at higher temperatures.

There has been recent remarkable progress in the "synthesis" of ultracold alkali dimers from samples of ultracold atoms, using photoassociation and magnetic tuning through Feshbach resonances [2]. From a chemical perspective, however, methods which enable the preparation of more diverse (e.g., polyatomic) cold molecular species in their vibronic ground states, such as Stark deceleration [4], electrostatic velocity selection [5], collisional cooling in crossed molecular beams [6], Zeeman deceleration [7], and sympathetic and laser cooling of ions [8], are particularly interesting. However, to date no cold-collision experiment combining these sources, and allowing for tunable collision energies, has been realized to our knowledge. The experimental challenge lies in detecting the small number of product molecules formed in a reactive-scattering experiment at the low densities of reactant molecules (typically $\sim 10^{8}$ molecules $\mathrm{cm}^{-3}$ ) which can be generated with these techniques [4-6].

This Letter reports for the first time an experimental method to study reactive collisions between ions and neutral molecules at very low temperatures which allows for both tunable collision energies and a variety of chemically diverse reaction partners. A quadrupole-guide velocity selector for the generation of cold neutral molecules [5] is combined with a source of spatially ordered laser-cooled ions ("Coulomb crystal") in an ion trap [9] and is applied to study the reaction of $\mathrm{CH}_{3} \mathrm{~F}$ and $\mathrm{Ca}^{+}$to form $\mathrm{CaF}^{+}$and $\mathrm{CH}_{3}$. The single-particle sensitivity achievable in reactive studies using Coulomb crystals $[8,10,11]$, as a consequence of the strong localization of the ions, is the key element of the present experiment to overcome the limitations imposed by the low reactant number densities.

In the experimental setup displayed in Fig. 1, cold $\mathrm{CH}_{3} \mathrm{~F}$ molecules are prepared by selecting the slowest molecules from the tail of the thermal velocity distribution of an effusive beam using a bent electric-quadrupole guide (bend radius $12.5 \mathrm{~mm}$, circular rod diameter $2 \mathrm{~mm}$, adjacent rod spacing $1 \mathrm{~mm}$ ) [5]. Molecules in low-field-seeking Stark states are repelled by the strong inhomogeneous fields near the electrodes towards the center of the quadrupole. Only the slowest molecules, whose kinetic energy is insufficient to escape the electrostatic potential inside the guide, are forced around the bend, whereas fast molecules are lost. The velocity selector is loaded from an effusive molecular beam exiting a $1.2 \mathrm{~mm}$ diameter capillary connected to a thermal $(T=298 \mathrm{~K})$ reservoir of $\mathrm{CH}_{3} \mathrm{~F}$ gas at 0.1 mbar. Differential pumping in three stages is used to maintain a working pressure of $<5 \times 10^{-10}$ mbar in the ion trap chamber. The flux and kinetic energy of the velocity-selected molecules is varied by tuning the voltages, $\pm V_{\mathrm{QUAD}}$, applied to the quadrupole rods. Figure 2 shows the unnormalized kinetic-energy distributions of the velocity-selected $\mathrm{CH}_{3} \mathrm{~F}$ molecules at different quadrupole-

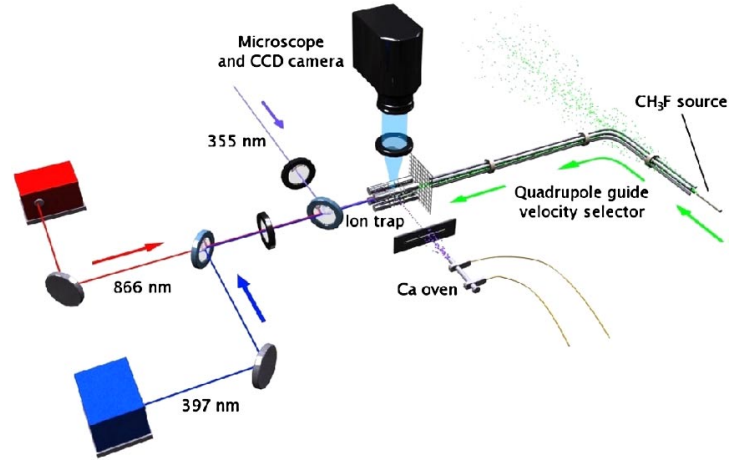

FIG. 1 (color online). Schematic representation of the experimental setup. 


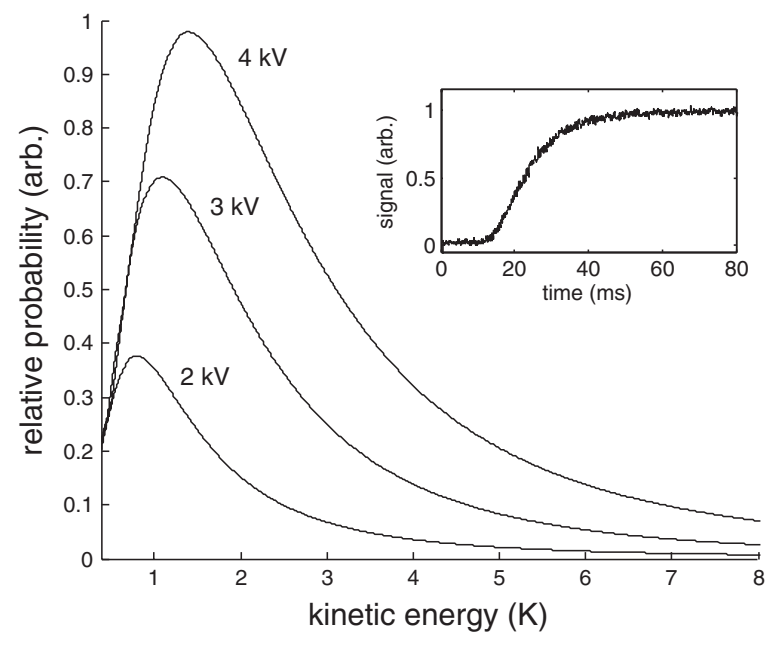

FIG. 2. Experimental kinetic-energy distributions of velocityselected $\mathrm{CH}_{3} \mathrm{~F}$ molecules as a function of the voltage $V_{\mathrm{QUAD}}$ applied to the quadrupole velocity filter. The inset shows the quadrupole mass analyzer signal as a function of time after applying a pulsed voltage $V_{\mathrm{QUAD}}= \pm 4.0 \mathrm{kV}$ to the quadrupole guide.

guide voltages; these were determined by pulsing the applied voltages and measuring the arrival times of the velocity-selected molecules using a quadrupole mass analyzer placed at the position of the ion trap center (inset in Fig. 2). The average kinetic energies $\bar{E}_{\text {kin }}$ range from $\bar{E}_{\text {kin }} / k_{B}=3.7(5) \mathrm{K}$ for the highest voltages used in the present study $( \pm 5.0 \mathrm{kV}$ ) to $2.0(5) \mathrm{K}$ for the lowest voltages $( \pm 2.0 \mathrm{kV})$. The rovibrational state populations of the guided molecules are only weakly affected by the velocity-filtering process and reflect the distribution of a room-temperature sample [12]. Note that both the widths of the kinetic-energy distributions and their integrated intensities (related to the flux of molecules exiting the guide) increase with increasing $\pm V_{\mathrm{QUAD}}$ as a result of successively faster molecules being transmitted through the velocity filter. The densities of cold molecules measured at the position of the ion trap range from 1.2(5) $\times$ $10^{5} \mathrm{~cm}^{-3}$, for $V_{\mathrm{QUAD}}= \pm 2.0 \mathrm{kV}$, to $1.1(5) \times 10^{6} \mathrm{~cm}^{-3}$ for $V_{\mathrm{QUAD}}= \pm 5.0 \mathrm{kV}$.

The velocity-selected $\mathrm{CH}_{3} \mathrm{~F}$ molecules were guided into the center of a linear Paul ion trap containing a Coulomb crystal of $\mathrm{Ca}^{+}$ions. The trap consists of four segmented stainless-steel rods (diameter $8 \mathrm{~mm}$ ) in a quadrupolar configuration [9]. $\mathrm{Ca}^{+}$was produced by nonresonant multiphoton ionization of a calcium-atom beam in the trap center using the $355 \mathrm{~nm}$ output of a frequency-tripled $\mathrm{Nd}$ :YAG laser. The ions are confined by the application of radiofrequency voltages (amplitude $V_{0-p}=150 \mathrm{~V}$, frequency $\Omega=2 \pi \times 3.8 \mathrm{MHz}$ ) to the central segments, and dc potentials $\left(V_{\text {end }}=1-5 \mathrm{~V}\right)$ to the end cap electrodes of the trap. $\mathrm{Ca}^{+}$Coulomb crystals were generated by Doppler laser cooling on the $4 s^{2} S_{1 / 2} \rightarrow 4 p^{2} P_{1 / 2}$ transition at $397 \mathrm{~nm}$ using the output of a narrow-bandwidth $(1 \mathrm{MHz})$ external-cavity-enhanced diode laser. An additional diode- laser beam at $866 \mathrm{~nm}$ was employed to repump population from the $3 d^{2} D_{3 / 2}$ level. The spontaneous emission from the ions was monitored through a microscope lens (magnification $10 \times$ ) and imaged onto an image-intensified CCD camera. Typical images are shown in Figs. 3(a) and 3(b) illustrating the characteristic ion spacing of $\sim 20 \mu \mathrm{m}$. The ions were shielded from the strong electric fields around the velocity selector by a grounded mesh placed between the exit of the quadrupole guide and the ion trap.

In order to control the collision energy of the reacting partners, the mean kinetic energy of the neutrals was changed by adjusting the quadrupole-guide voltage. The average kinetic energy of the ions remained fixed for a given set of trapping and cooling conditions and was determined by a comparison of the fluorescence images with molecular dynamics (MD) simulations [12]. The effective kinetic energy of the ions, taking into account the contribution of the thermal as well as the fast oscillating micromotion of the ions in the $\mathrm{rf}$ field [13], is a function of the crystal size and ranges from $\bar{E}_{\mathrm{kin}, \mathrm{eff}} / k_{B} \approx 400 \mathrm{mK}$ for the smallest to $\approx 3.5 \mathrm{~K}$ for the largest crystals generated in the present study [12].

The progress of the chemical reaction between the lasercooled $\mathrm{Ca}^{+}$ions and the translationally cold $\mathrm{CH}_{3} \mathrm{~F}$ molecules was monitored by measuring the decrease of the number of $\mathrm{Ca}^{+}$ions observed in the fluorescence images as a function of the time of exposure to the flow of $\mathrm{CH}_{3} \mathrm{~F}$.

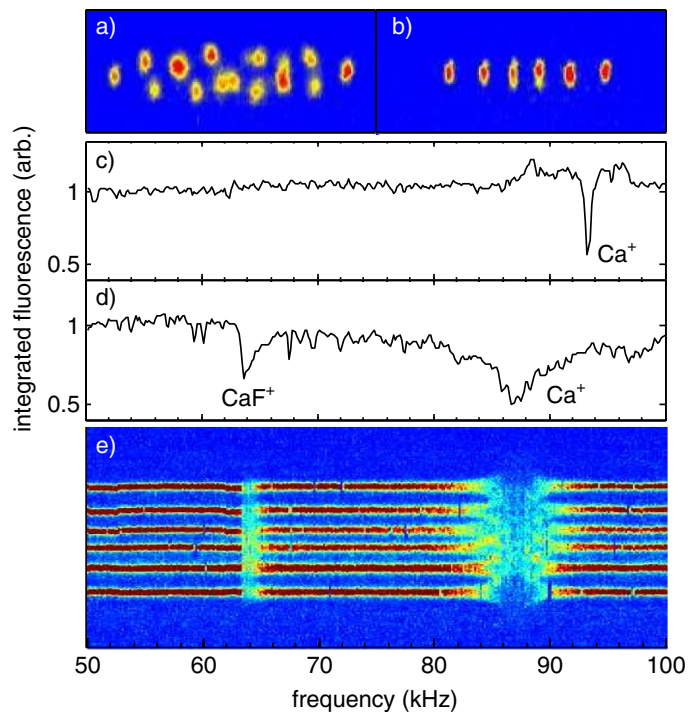

FIG. 3 (color online). False-color fluorescence images of a small (15-ion) $\mathrm{Ca}^{+}$Coulomb crystal (a) before and (b) after the chemical reaction with velocity-selected $\mathrm{CH}_{3} \mathrm{~F}$ molecules. (c),(d) Resonant-excitation mass spectra of the Coulomb crystals in (a) and (b), measured by monitoring the integrated fluorescence of the $\mathrm{Ca}^{+}$ions as a function of an additional $\mathrm{rf}$ drive frequency applied to the end caps of the ion trap. The decrease of the $\mathrm{Ca}^{+}$fluorescence at the position of the resonances in (d) is caused by a reversible melting of the crystal as can be seen in (e), which shows the sequence of fluorescence images of the crystal in (b) (rotated by $90^{\circ}$ ) recorded during the mass scan. 
Figure 3(a) shows the fluorescence image of a 15 -ion $\mathrm{Ca}^{+}$ Coulomb crystal, while Fig. 3(b) shows the same crystal after $80 \mathrm{~min}$ exposure to translationally cold $\mathrm{CH}_{3} \mathrm{~F}$. The quadrupole guide was held at $V_{\mathrm{QUAD}}= \pm 2.0 \mathrm{kV}$ corresponding to $\bar{E}_{\text {kin }} / k_{B}=2.0 \mathrm{~K}$ and the average effective kinetic energy of the 15-ion crystal was determined to be $\bar{E}_{\text {kin,eff }} / k_{B} \approx 450 \mathrm{mK}$, yielding an average collision energy of $\bar{E}_{\text {coll }} / k_{B}=1.3 \mathrm{~K}$. The observed removal of fluorescing $\mathrm{Ca}^{+}$ions over time is attributed to reactive collisions with velocity-selected $\mathrm{CH}_{3} \mathrm{~F}$ molecules. By comparison, in a control experiment, in which the flow of cold neutrals was switched off, only two ions were lost from a similarly sized crystal over the same period of time, probably because of reactive collisions with backgroundgas molecules. The importance of trap-loss processes of $\mathrm{Ca}^{+}$ions caused by elastic collisions was studied by exposing the Coulomb crystal to $5 \times 10^{-9}$ mbar of nonreactive $\mathrm{ND}_{3}$ background gas. The loss rates were found to be negligible under the present experimental conditions.

The $\mathrm{Ca}^{+}(4 s)+\mathrm{CH}_{3} \mathrm{~F}$ reaction is strongly exothermic $(\Delta E=-1.05 \mathrm{eV}$ [14]). An undetermined fraction of this energy goes into the internal degrees of freedom of the products, while most of the kinetic energy is carried away by the lighter $\mathrm{CH}_{3}$ radicals; thus the nonfluorescing product $\mathrm{CaF}^{+}$ions are retained in the ion trap (minimum trap depth of $\approx 1.2 \mathrm{eV}$ ) and sympathetically cooled by the remaining laser-cooled $\mathrm{Ca}^{+}$ions to form a bicomponent Coulomb crystal. This is established (postreaction) by resonant-excitation mass spectra of the ions in the trap, displayed in Figs. 3(c) and 3(d), recorded by monitoring the total $\mathrm{Ca}^{+}$fluorescence as a function of the frequency of an additional $\mathrm{rf}$ voltage (amplitude $V_{\text {drive }}=3 \mathrm{mV}$ ) applied to the trap end caps [15]. When the rf drive is resonant with the frequency of the center-of-mass motion of one of the species in the trap, the ions are driven into high amplitude oscillation and the absorption frequency of the laser-cooled ions is Doppler shifted out of resonance with the coolinglaser frequency causing a decrease of the fluorescence intensity. The mass spectrum of the Coulomb crystal before exposure to $\mathrm{CH}_{3} \mathrm{~F}$, shown in Fig. 3(c), only exhibits a single feature at $93.5 \mathrm{kHz}$ corresponding to the excitation of the motional resonance of the $\mathrm{Ca}^{+}$ions in a pure crystal, whereas the mass spectrum of the crystal in Fig. 3(d), recorded postreaction, shows two resonances attributed to the excitation of $\mathrm{CaF}^{+}$and $\mathrm{Ca}^{+}$. The broadening and shift of the resonance features towards lower frequencies compared to a single-species crystal are induced by the spacecharge drag of the different ion species on each other and are indicative of the tight packing of the ions in a bicomponent crystal [15]. Thus, the mass spectra provide conclusive evidence that reactive collisions between the cold ions and neutrals occur inside the ion trap and that at least some of the product ions are trapped and sympathetically cooled.

Recent studies have demonstrated that the ion density in large Coulomb crystals ( $\geq 100$ ions) is approximately con- stant $[16,17]$, and so the volume of the $\mathrm{Ca}^{+}$crystal is proportional to the number of ions, and the decrease of the volume is a direct measure of the of extent of reaction of $\mathrm{Ca}^{+}$ions. Because of the radial symmetry of the effective potential in the ion trap, the Coulomb crystals are cylindrically symmetric and the crystal volumes are easily determined from the fluorescence images [17]. Figure 4(a) shows a logarithmic plot of the apparent volume (i.e., that occupied by $\mathrm{Ca}^{+}$) of a 250-ion crystal as a function of reaction time with $\mathrm{CH}_{3} \mathrm{~F}$. The rate of the decrease of the crystal volume $V$ is consistent with a pseudo-first-order integrated rate law of the form

$$
V(t)=V(0) \exp \left\{-N_{\mathrm{CH}_{3} \mathrm{~F}} k_{\mathrm{bi}} t\right\},
$$

where $N_{\mathrm{CH}_{3} \mathrm{~F}}$ denotes the number density of $\mathrm{CH}_{3} \mathrm{~F}$ molecules and $k_{\mathrm{bi}}$ is the bimolecular rate constant of the reaction; the pseudo-first-order behavior arises because the flux of $\mathrm{CH}_{3} \mathrm{~F}$ molecules through the reaction region is constant with time. The bimolecular rate constant $k_{\mathrm{bi}}$ is determined by a fit of Eq. (1) to the crystal volumes measured as a function of reaction time. At $V_{\mathrm{QUAD}}= \pm 3.0 \mathrm{kV}$, we find $k_{\mathrm{bi}}=1.3(6) \times 10^{-9} \mathrm{~cm}^{3} \mathrm{~s}^{-1}$. The experimental error predominantly reflects the uncertainty in the determination of the $\mathrm{CH}_{3} \mathrm{~F}$ flux.

The room-temperature value of the rate constant $k_{\mathrm{bi}}=$ $4.2(4) \times 10^{-10} \mathrm{~cm}^{3} \mathrm{~s}^{-1}$ which was determined by admitting $\mathrm{CH}_{3} \mathrm{~F}$ gas into the ion trap chamber through a leak valve is consistent with the results of previous studies [14]. In Fig. 4(b) the collision-energy dependence of $k_{\mathrm{bi}}$ is presented. The average collision energy was varied over
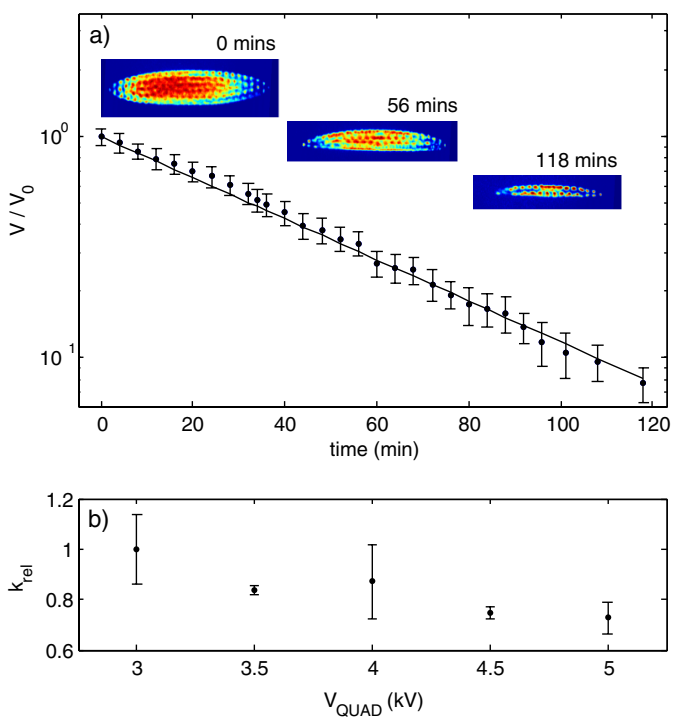

FIG. 4 (color online). (a) Logarithmic plot of the ratio of volumes $V(t) / V(0)$ occupied by $\mathrm{Ca}^{+}$ions of a Coulomb crystal of 250-ion crystal as a function of the reaction time according to the pseudo-first-order rate law Eq. (1) with $V_{\mathrm{QUAD}}= \pm 3.0 \mathrm{kV}$. (b) Normalized reaction rates $k_{\text {rel }}=k_{\mathrm{bi}} / k_{\mathrm{bi}}\left(V_{\mathrm{QUAD}}= \pm 3.0 \mathrm{kV}\right)$ as a function of $V_{\mathrm{QUAD}}$. The error bars reflect the $1 \sigma$ uncertainty of four measurements performed for each value of $V_{\mathrm{QUAD}}$. 
the range $\bar{E}_{\text {coll }} / k_{B}=2.6-3.2 \mathrm{~K}$ by tuning $V_{\mathrm{QUAD}}$ from $\pm 3.0 \mathrm{kV}$ to $\pm 5.0 \mathrm{kV}$ and the rate constant values presented are normalized with respect to the value at $V_{\mathrm{QUAD}}=$ $\pm 3.0 \mathrm{kV}$. The values for $k_{\mathrm{bi}}$ show a slight inverse dependence on the collision energy, in line with ion-molecule adiabatic capture theories $[12,18]$. A detailed interpretation of these low-temperature dynamics will require accurate global potential energy surfaces for the $\mathrm{Ca}^{+}+\mathrm{CH}_{3} \mathrm{~F}$ system which are currently unavailable. It is expected that the reaction path is electronically nonadiabatic [14]. The values of the rate constants also show a dependence on the intensity and detuning of the cooling laser, indicating the participation of excited states of $\mathrm{Ca}^{+}$in the reaction, and a dependence on the size and shape of the crystals, attributed to different kinetic-energy distributions of the ions; these effects will be discussed in a future publication [12].

In conclusion, we have demonstrated the first combination of two different experimental techniques for the generation of cold atoms and molecules to study cold reactive collisions. The present experimental setup enables the study of ion-molecule reactions down to collision energies of $\bar{E}_{\text {coll }} / k_{B} \geq 1 \mathrm{~K}$ with single-particle sensitivity. The lower limit is about 1 order of magnitude smaller than currently achieved using the CRESU technique (in which the reactants are thermally equilibrated in a helium buffer gas cooled in a Laval nozzle expansion [19]), which has thus far been the standard method to study chemical reactions at low temperatures. Previous studies of ion-molecule reactions performed around $1 \mathrm{~K}$ in supersonic jets were limited to a very narrow temperature range of only a few Kelvin [20]. With the present method, however, it is in principle possible to access a wide range of collision energies by varying the velocity distribution of the neutrals, changing the size and aspect ratio of the Coulomb crystals and heating up the ions in the trap using an additional rf drive [12]. The present technique is not limited to the study of reactions with laser-cooled atomic ions. Reactive collisions with a variety of molecular ions can be investigated using molecular Coulomb crystals formed by sympathetic cooling $[8,11]$. Hence, the technique presented here can be regarded as a general approach towards studying lowenergy reactive collisions between molecular ions and polar neutrals and is well suited to investigate reactions of astrophysical importance at the low interstellar temperatures [21].

Two further developments of the current setup are envisaged. First, even lower collision energies $(\sim \mathrm{mK})$ could be achieved by combining the Coulomb crystal apparatus with a Stark decelerator [4]. At such temperatures only a few angular momentum partial waves will contribute to the reaction, compared to $\sim 50$ in the present experiment. Second, the preparation of both reactant species in a well-defined internal state is desirable in order to study fully state-selected cold ion-molecule reactions. Cold neutrals prepared by Stark deceleration are generically state selected, while state-selected molecular ions in a Coulomb crystal could be prepared by photoionization $[22,23]$. Work in both directions is currently being performed in our laboratory.

We thank Professor Michael Drewsen (Aarhus) for his help in the development of the ion-cooling apparatus and Dr. Peter Blythe (Sussex) for helpful discussions. This work is financially supported by EPSRC.

*stefan.willitsch@chem.ox.ac.uk

†tim.softley@chem.ox.ac.uk

[1] P. F. Weck and N. Balakrishnan, Int. Rev. Phys. Chem. 25, 283 (2006).

[2] J. M. Hutson and P. Soldán, Int. Rev. Phys. Chem. 25, 497 (2006).

[3] T. Stoecklin and Ph. Halvick, Phys. Chem. Chem. Phys. 7, 2446 (2005).

[4] H. L. Bethlem and G. Meijer, Int. Rev. Phys. Chem. 22, 73 (2003).

[5] S. A. Rangwala, T. Junglen, T. Rieger, P. W. H. Pinkse, and G. Rempe, Phys. Rev. A 67, 043406 (2003).

[6] M.S. Elioff, J. J. Valentini, and D. W. Chandler, Science 302, 1940 (2003).

[7] N. Vanhaecke, U. Meier, M. Andrist, B. H. Meier, and F. Merkt, Phys. Rev. A 75, 031402 (2007).

[8] K. Molhave and M. Drewsen, Phys. Rev. A 62, 011401 (2000).

[9] M. Drewsen, I. Jensen, J. Lindballe, N. Nissen, R. Martinussen, A. Mortensen, P. Staanum, and D. Voigt, Int. J. Mass Spectrom. 229, 83 (2003).

[10] T. Baba and I. Waki, J. Chem. Phys. 116, 1858 (2002).

[11] B. Roth, P. Blythe, H. Wenz, H. Daerr, and S. Schiller, Phys. Rev. A 73, 042712 (2006).

[12] S. Willitsch, M. Bell, A. Gingell, J. Oldham, and T.P. Softley (to be published).

[13] D. J. Berkeland, J. D. Miller, J. C. Bergquist, W. M. Itano, and D. J. Wineland, J. Appl. Phys. 83, 5025 (1998).

[14] J. N. Harvey, D. Schröder, W. Koch, D. Danovich, S. Shaik, and H. Schwarz, Chem. Phys. Lett. 273, 164 (1997).

[15] B. Roth, P. Blythe, and S. Schiller, Phys. Rev. A 75, 023402 (2007).

[16] L. Hornekær, N. Kjærgaard, A. M. Thommesen, and M. Drewsen, Phys. Rev. Lett. 86, 1994 (2001).

[17] U. Fröhlich, B. Roth, and S. Schiller, Phys. Plasmas 12, 073506 (2005).

[18] D. C. Clary, Chem. Phys. Lett. 232, 267 (1995).

[19] I. W. M. Smith, Angew. Chem., Int. Ed. Engl. 45, 2842 (2006).

[20] T. L. Mazely and M. A. Smith, Chem. Phys. Lett. 144, 563 (1988).

[21] D. Smith and P. Spanel, Mass Spectrom. Rev. 14, 255 (1995).

[22] S. R. Mackenzie and T. P. Softley, J. Chem. Phys. 101, 10609 (1994).

[23] S. Willitsch and F. Merkt, Int. J. Mass Spectrom. 245, 14 (2005). 